\title{
Nordiques
}

39 | 2020

Varia

\section{Du Vennamoïsme au parti des Finlandais : généalogie du populisme en Finlande}

Nathalie Blanc-Noël

\section{OpenEdition}

Journals

Édition électronique

URL : http://journals.openedition.org/nordiques/587

DOI : $10.4000 /$ nordiques.587

ISSN : 2777-8479

Éditeur :

Association Norden, Bibliothèque de Caen la mer

Référence électronique

Nathalie Blanc-Noël, « Du Vennamoïsme au parti des Finlandais : généalogie du populisme en

Finlande », Nordiques [En ligne], 39 | 2020, mis en ligne le 01 novembre 2020, consulté le 13 mars

2021. URL : http://journals.openedition.org/nordiques/587 ; DOI : https://doi.org/10.4000/nordiques.

587

Ce document a été généré automatiquement le 13 mars 2021.

Nordiques 


\title{
Du Vennamoïsme au parti des Finlandais : généalogie du populisme en Finlande
}

\author{
Nathalie Blanc-Noël
}

\section{Introduction}

1 Au début des années 2000, la Finlande passait pour une exception, car elle était l'un des rares pays européens échappant à la montée du populisme de droite radicale ${ }^{1}$ en Europe. Ce courant politique correspond à la définition donnée par le politologue suédois Jens Rydgren : les partis populistes de droite radicale «partagent un noyau de xénophobie ethnonationaliste et de populisme anti-establishment» s'accompagnant dans leurs plateformes politiques d'un «autoritarisme socioculturel général qui met l'accent sur des thèmes tels que la loi et l'ordre et les valeurs familiales $»^{2}$. Pourtant, le Parti des Finlandais (Perussuomalaiset - PS ${ }^{3}$ ), un parti nationaliste, souverainiste et anti-immigration, n'a cessé de progresser au cours de ces années 2000. Aux élections parlementaires de 2011, que les Finlandais nomment « la grosse secousse " », il est arrivé en $3^{\text {ème }}$ place avec $19 \%$ des voix, dans un système politique très fragmenté, où un parti ne peut généralement espérer dépasser les $25 \%$. Aux législatives de 2015, il, est arrivé en $2^{\text {ème }}$ position et a participé à la coalition gouvernementale avec le parti de la Coalition nationale et du Centre. Après une scission et le départ de son leader, Timo Soini, pour une nouvelle formation appelée Réforme bleue (Sininen Tulevaisuus), PS a tout de même obtenu une troisième place à l'élection présidentielle de 2018 (avec $6,9 \%$ des voix $)^{5}$, puis il a fortement remonté aux législatives de 2019 où il est arrivé second, au coude à coude avec les Sociaux-Démocrates (17,48\% des voix pour PS/ $17,73 \%$ pour les Sociaux-démocrates) ${ }^{6}$. Cette fois cependant, les Vrais finlandais, dirigés par Jussi Halla-Aho, représentant la ligne dure du parti, ne participent pas à la coalition gouvernementale formée en 2019, les Sociaux-démocrates, les Verts et la Ligue de gauche ayant annoncé à l'avance ne pas souhaiter former de coalition avec eux ${ }^{7}$. 
2 A première vue, le populisme finlandais est un phénomène paradoxal: s'il a d'indéniables traits du populisme d'extrême-droite, sous le leadership de Timo Soini, il siégeait au centre de l'Eduskunta, alors même qu'il a été membre fondateur du groupe «Europe libertés démocratie » au Parlement européen, avec le UKIP britannique et le Parti du peuple danois. Après avoir un temps siégé au Groupe «Conservateurs et réformistes européens ", aujourd'hui, sous le leadership de Jussi Halla-Aho, il est membre du groupe identité et démocratie, avec notamment la Ligue du nord italienne, le FPÖ autrichien, le parti du peuple danois et le Rassemblement national. A l'Eduskunta, il siège à droite depuis les législatives de 2019. Autre paradoxe, le Parti des Finlandais est un parti anti-immigration dans un des pays européens les moins concernés par ce phénomène... Pour comprendre ces paradoxes, nous nous livrerons à une généalogie du parti, avant d'en étudier le programme. Mais auparavant il nous faut souligner deux particularités de la vie politique finlandaise héritées de l'histoire du pays, qui ont été des préconditions propices à l'avènement du populisme: l'héritage culturel et l'héritage de la guerre froide.

\section{Les préalables historiques au populisme finlandais}

3 La Finlande a fait partie du royaume de Suède pendant plus de cinq siècles ${ }^{8}$ avant de devenir un grand-duché de l'empire russe en 1809. Elle gagna son indépendance en 1917, puis une guerre civile opposant « Rouges » et «Blancs » déchira le pays. Ce conflit politique s'inscrivait dans le prolongement d'un double clivage social et linguistique'. $\mathrm{Au}$ milieu du $19^{\mathrm{ème}}$ siècle, l'étroit segment de population situé en haut de la pyramide sociale était suédophone, de même que les classes moyennes urbaines (commerçants, artisans), dans une Finlande encore très majoritairement rurale. L'éveil du nationalisme finlandais se fit au cours de la période où le pays fut un grand-duché de l'empire russe. Il s'appuya sur les institutions héritées de la période suédoise (la Finlande au sein de l'empire russe conserva ses lois et ses infrastructures, ce qui lui permit notamment de développer un parlementarisme moderne). Au cœur du jeune nationalisme finlandais était la question linguistique: le développement du finnois accompagnait le projet de création d'un État-nation indépendant ${ }^{10}$. Á la fin du $19^{\text {ème }}$ siècle, une élite finnophone se développa (alors que beaucoup de suédophones émigraient vers les Amériques). Le clivage linguistique et social se prolongea en politique ${ }^{11}$. Au cours de la guerre civile, les suédophones furent surreprésentés dans les rangs des Blancs, opposés à la domination de la Russie ; les Blancs l'emportèrent, sous la direction du Maréchal Mannerheim, et la Finlande put devenir une république (dont la constitution assurait la garantie des droits des deux groupes linguistiques). Lénine, qui avait espéré une révolution marxiste dans le pays, reconnut finalement l'indépendance de la Finlande le 31 décembre 1917. Au cours de la $2^{\mathrm{e}}$ guerre mondiale, la Finlande s'opposa à deux reprises à l'URSS (Guerre d'hiver puis Guerre de continuation). Elle fut défaite, mais ces épisodes ont cimenté le jeune nationalisme finlandais, et gommé certaines divisions qui avaient déchiré le pays durant de la guerre civile $^{12}$.

4 Pour autant, des enquêtes d'opinion menées au cours des années 1970 et $1990^{13}$ ont montré que la population finnophone partageait des valeurs plus conservatrices, plus nationalistes, et était plus attachée aux valeurs traditionnelles que la population suédophone. De plus, les finnophones étaient plus critiques envers les hommes 
politiques, les partis et les fonctionnaires que les suédophones ${ }^{14}$. Or, le discours du populisme finlandais s'adresse prioritairement à cette population. PS a ainsi tenté de ranimer la vieille querelle linguistique en proposant de supprimer l'enseignement obligatoire du suédois à l'école, en reprenant l'argumentaire du tout début du vingtième siècle, selon lequel le suédois apparaissait comme un élément étranger argument qui s'accompagnait à l'époque de l'idée que ceci constituait un risque pour la sécurité d'un jeune État-nation. Le nom choisi par l'actuel parti populiste est d'ailleurs similaire à celui du mouvement des "Vrais Finlandais", un club politique créé à Helsinki en 1923, et dont le but était d'abolir le bilinguisme, de réduire le statut du suédois à celui de dialecte régional et de réduire dans le même temps les fonds publics alloués à la culture suédoise ${ }^{15}$, revendications reprises à l'identique par le parti contemporain.

5 Après la Seconde Guerre mondiale, la Finlande fut le seul pays limitrophe de l'URSS à ne pas être incorporé au bloc de l'Est, mais elle dut signer en 1948 un Traité d'amitié, de coopération et d'assistance mutuelle avec Moscou, qui l'aurait obligée en cas d'attaque à combattre à ses côtés. C'est pourquoi, pendant la guerre froide, la vie politique finlandaise s'est déroulée en tenant compte de la proximité de l'URSS, dans un climat d'autocensure, situation que les étrangers ont appelé «finlandisation». De plus les traités de paix de Moscou (1944) et de Paris (1947) interdisaient les partis fascistes, d'extrême-droite ainsi que les organisations racistes en Finlande.

6 Le tout-puissant président Kekkonen, Président de la République de 1956 à 1981, était personnellement en relation avec le Kremlin. Au motif de la situation précaire dans laquelle se trouvait son pays, il réussit à dominer le jeu politique, choisissant ministres et Premiers ministres comme le permettait la Constitution (en respectant le résultat des élections mais en tenant compte, à certains moments difficiles, des préférences de Moscou $)^{16}$. En matière de politique intérieure, et plus encore en matière de politique étrangère, le consensus et l'auto-censure étaient considérés comme indispensables pour la sécurité du pays. Le grand public finit par laisser l'exercice d'un jeu politique biaisé et dangereux aux élites. Á la fin de la guerre froide, la situation changea et la Finlande passa " d'une culture silencieuse, régulée et basée sur le self-control à une culture bruyante et tapageuse et d'une certaine manière "dirigée par le peuple" 17 ». La scène politique finlandaise est aujourd'hui très différente de ce qu'elle était dans les années 80 : la parole s'y est véritablement libérée, et son bouillonnement se prolonge jusque sur Internet (Les Finlandais étant comparativement très connectés ${ }^{18}$ ). Le populisme finlandais a particulièrement bénéficié de ce moyen d'expression, ainsi que de la libération de la parole politique qui s'est produite à ce moment-là.

\section{L'ascension du populisme en Finlande}

7 Le populisme était déjà présent sur la scène politique du pays au cours de la guerre froide; PS est l'héritier d'un mouvement plus ancien, le "vennamoïsme ». Dans les années 60 , le pays se modernisa, s'industrialisa, l'exode rural augmenta, ainsi que la pauvreté dans les campagnes et le mécontentement des classes rurales. Le parti du Centre, parti agrarien qui avait traditionnellement représenté les classes paysannes, perdit du terrain et nombre de ses électeurs se tournèrent vers un nouveau venu, le Parti rural finlandais (SMP), issu d'une faction du Parti du centre. Ce parti, fondé par Veikko Vennamo en 1966, était typiquement populiste. Très lié à la personne de son 
fondateur, un personnage au verbe haut, le " vennamoïsme » sut exploiter la déception des électeurs ruraux vis-à-vis du Parti du centre et la désorientation liée à la modernisation $d u$ pays pour prôner un retour aux valeurs paysannes. Vennamo critiquait les élites, en particulier politiques, qu'il appelait «les seigneurs délinquants ». Ce pourfendeur de la corruption et du népotisme s'en prenait aussi aux bureaucrates, aux experts de tout poil et aux intellectuels. Vennamo visait en particulier la toute-puissance du Président Kekkonen - affirmant que son pays était devenu une véritable "Kekkoslovaquie ${ }^{19}$. Il opposait aux élites le petit peuple, honnête et travailleur, le peuple "oublié » mais plein de bon sens. Il défendait des valeurs conservatrices, chrétiennes et la culture finnoise. Les succès du SMP furent fluctuants. Son meilleur score fut de $10 \%$ en 1970. Il entra deux fois au gouvernement dans les années 80 puis son succès diminua. Les années 90 furent marquées par une terrible récession, et le Parti du Centre reprit la main.

8 Lorsque le vennamoïsme s'effondra, quatre de ses membres fondèrent le Parti des Finlandais (PS Perussuomalaiset). Parmi eux, Timo Soini, qui avait été le dernier secrétaire du parti rural, devint président de PS en 1997. La progression de ce parti fut régulière : il entra au Parlement en 2003. Aux élections européennes de 2009, il fit un peu moins de $10 \%$ et aux élections parlementaires de 2011 (la " grosse secousse »), PS obtint $19 \%$ des voix ( $3^{\text {ème }}$ parti en nombre de voix). Mais Soini refusa d'entrer dans la coalition gouvernementale, au motif que cela l'aurait obligé à soutenir une politique européenne avec laquelle il était en désaccord. Le chercheur Tapio Raunio ${ }^{20}$ a émis l'hypothèse qu'à ce motif « officiel » s'ajoutait une autre raison : le parti connaissait des dissensions internes, notamment au sujet de l'immigration : il abritait en effet en son sein une "faction » anti-immigration, menée par Jussi Halla-Aho et représentant 5,5\% du total des candidats en 2011 - soit une quinzaine de députés après cette élection. Mais une autre explication, de nature plus stratégique, peut être avancée : 34 des 39 députés de PS élus en 2011 n'avaient pas encore l'expérience de la vie parlementaire ni celle du gouvernement, et le souvenir de l'expérience du SMP était encore vivace : ce parti se mit à décliner lorsqu'il participa à des gouvernements. Ses scores baissèrent ensuite, mais aux européennes de 2014, ils remontèrent à presque $13 \%$ ( $2^{\text {ème }}$ position). Aux législatives de 2015, ils conservèrent cette position avec $17,65 \%$ des voix ( $2^{\text {ème }}$ position). PS entra alors au gouvernement. Timo Soini obtint le portefeuille des Affaires étrangères et PS obtint aussi ceux de la Justice et de l'Emploi, de la Défense et des Affaires sociales. Ces portefeuilles correspondaient aux principaux thèmes développés par le parti: l'Europe, la sécurité, l'aide aux démunis et bien sûr l'immigration.

9 L'ascension de PS repose sur des facteurs très comparables à ceux ayant favorisé le vennamoïsme: PS s'est développé lors d'une mutation de l'économie; cette fois le passage d'une économie industrialisée à une économie basée sur les technologies de l'information. Il a bénéficié à la fois d'un désaveu du parti du Centre et du parti Socialdémocrate, à une période où l'économie finlandaise était en difficulté, les suites de la récession des années 90 se faisant toujours sentir ${ }^{21}$. Depuis le milieu des années 90 , le creusement des revenus a été plus rapide en Finlande que dans les autres pays de l'OCDE ${ }^{22}$ et les statistiques montrent que PS a fait son lit de la montée du chômage. Il a su rallier les déceptions et les craintes éprouvées par certains citoyens face aux changements sociétaux: rigueur économique, réforme de l'État-providence, entrée dans l'Union européenne... puis crise des migrants. 
10 La figure du leader est particulièrement déterminante dans la trajectoire et l'ascension des partis populistes. C'est Timo Soini qui a structuré le parti des Finlandais sur l'héritage du vennamoïsme, tout en le modernisant. Soini est un leader charismatique, avec une personnalité simple, aimant le contact avec les gens, la culture populaire. Tout comme Vennamo avant lui, il utilise un ton radicalement différent de celui des autres politiciens, qui a fait son succès dans les médias. Sa popularité est très grande : aux élections municipales de 2008, aux européennes de 2009 et aux législatives de 2011, il a été le candidat ayant obtenu le plus de voix à titre individuel ${ }^{23}$. Employant une rhétorique simple et dénonçant les politiciens « classiques », il a porté la parole de tous les déçus de la politique, en particulier après les gouvernements de coalitions « arc-enciel » menés par Paavo Lipponen (1995 et 2003) ${ }^{24}$. Des sondages ont montré que, dans les années 2000, les Finlandais en étaient arrivés à confondre les partis politiques ${ }^{25}$. Soini a également su exploiter les scandales sur le financement des partis, qui ont particulièrement affecté le parti du Centre ${ }^{26}$ qui perdit des voix au profit de PS. Cela lui a permis de se positionner comme le seul parti différent de tous les autres. Il a aussi exploité la montée de l'immigration, qui est un phénomène très récent en Finlande. Enfin, le parti a bénéficié de son opposition constante à l'Europe et aux plans de sauvetage des pays surendettés - Portugal, Irlande, Grèce - avec l'argument que les Finlandais ne devaient pas payer pour les dettes des autres Etats.

11 Mais une fois au gouvernement, Soini n'a pu tenir toutes ses promesses : il a choisi le portefeuille des Affaires étrangères (alors que celui de ministre des Finances lui revenait de droit). Or, ce poste, l'amenant à voyager et à rencontrer les grands de ce monde, le faisait appartenir à un univers qu'il avait dénigré dans ses campagnes. Pire, il fit profil bas sur les questions européennes, ce qui fut certainement la première trahison vis-à-vis des électeurs qui l'avaient soutenu sur cette question - la seconde étant la crise des migrants au cours de laquelle la Finlande accueillit sa part de réfugiés. Quelques mois après l'entrée au pouvoir de PS, un sondage indiquait en octobre 2015 que près de $60 \%$ des électeurs de PS auraient reconsidéré leur vote si les élections s'étaient tenues alors... Au sein du parti, l'opposition entre l'aile modérée représentée par Soini et l'aile dure, ouvertement raciste, représentée par Jussi Halla-Aho se fit plus rude. Au moment de la réélection du leader du parti, Soini annonça qu'il ne serait pas candidat et Halla-Aho l'emporta. Soini quitta le parti, entraînant avec lui les modérés, qui formèrent un nouveau parti, «Réforme bleue » (Sininen Tulevaisuus). C'est ce parti qui siégea au gouvernement Sipilä jusqu'à son terme, dans une situation étrange, puisqu'il n'avait pas été élu. Réforme bleue se dit conservateur et plus modéré que PS ; il critique les autres partis, souhaitant respecter les traditions tout en ayant des visées réformatrices en particulier pour maintenir l'État-providence. Il a récemment évolué vers des positions plus dures vis-à-vis de l'immigration. Pour l'instant, c'est un parti aux résultats très modestes, qui n'a pu présenter aucun candidat à l'élection présidentielle de 2018. Aux législatives de 2019 (en l'absence de Soini, celui-ci ayant quitté la vie politique) il n'a obtenu aucun siège au parlement. En revanche, PS, écarté du pouvoir, a rapidement reconstitué ses forces jusqu'à se hisser à la $2^{\text {ème }}$ place de l'élection législative de 2019. Certes, aux élections européennes, son score n'a pas été aussi brillant, mais il est tout de même arrivé en $4^{\text {ème }}$ position avec des candidats dont le profil n'était pas «facile» (mais néanmoins séduisant pour une partie de l'électorat de PS) : Laura Huhtasaari est une enseignante créationniste, et Teuvo Hakkarainen, auteur de propos racistes, a été condamné pour harcèlement sexuel... 


\section{L'idéologie du parti des Finlandais : un populisme de droite radicale}

à quelques échecs essuyés par son prédécesseur, mais globalement, le cap est le même. Les Vrais Finlandais sont un parti nationaliste, visant la défense de la culture finlandaise dans le monde, et s'adressant au «vrai peuple» finlandais, fait de travailleurs modestes, mais pleins de bon sens. Le programme de principe de 2018 débutait ainsi : «L'accent est mis sur la valeur des citoyens finlandais ordinaires et leur rôle et leur voix dans la politique, l'économie et la culture de la Finlande ${ }^{27} \ldots$ ». Soini inscrivait clairement son message dans la tradition du nationalisme finlandais, qui reposait sur la question linguistique. Celle-ci figurait en effet dans les quatre points principaux du programme électoral de 2015, aux côtés de la politique économique, de la politique de sécurité et de la politique d'immigration ${ }^{28}$. Soini avait formulé l'objectif majeur de supprimer le bilinguisme de la Constitution ${ }^{29}$, d'abolir l'obligation de suivre des cours de suédois à l'école et de réduire les émissions en suédois des télévisions publiques, mais aussi de faciliter l'apprentissage du finnois pour les immigrés. Le parti organisa une pétition contre l'enseignement obligatoire du suédois et recueillit les 50000 signatures nécessaires pour inscrire la question à l'ordre du jour de l'Eduskunta. Mais les députés votèrent massivement contre cette proposition (134 voix contre 48). Depuis ce désaveu, le parti semble avoir pris quelques distances avec le nationalisme linguistique.

Le nationalisme de PS s'accompagne d'une prise de position contre le multiculturalisme, que PS présente non pas comme un fait social, mais comme une idéologie dangereuse ou comme une idée à la mode ${ }^{30}$. Dans le programme pour les législatives de 2007, on pouvait lire par exemple «Nous n'accepterons jamais les décisions qui impliquent de contraindre la population autochtone à sacrifier ses propres traditions pour s'accommoder à des cultures étrangères ${ }^{31}$ \%. Il était expliqué que si les immigrés ne s'intègrent pas à la société, mais constituent des sous-cultures, cela finirait par menacer la culture finnoise... Sous Halla-Aho, la charge contre le multiculturalisme est formulée différemment, et non sans ambiguité. PS semble reconnaître une certaine diversité culturelle en Finlande (les suédophones et les Sames étant des électeurs potentiels):

« Le cœur de la culture finnoise n'est pas constitué d'une seule tradition, attitude, opinion ou d'un «style » unique - c'est une combinaison de la langue, de l'histoire, des coutumes, des valeurs et des symboles » [...] «Un Finlandais sait ce que cela signifie d'être finlandais, et être finlandais «c'est reconnaître quelque chose dans l'esprit ${ }^{32}$.

15 La lutte contre l'immigration au nom de la défense du peuple finlandais, dans sa culture, mais aussi en ce qui concerne son bien-être matériel, constitue le cœur de l'idéologie du parti. Ce qui est paradoxal, c'est que le nombre d'immigrés en Finlande est l'un des plus faibles en Europe, même s'il est en augmentation: en 2018, selon 
Statistics Finland, $7 \%$ de la population était née à l'étranger (par ordre d'importance : en Russie, Estonie, Suède, Irak, Somalie...). PS est sans doute conscient du fait qu'il s'est positionné comme un parti anti-immigration dans un pays où le phénomène est longtemps resté quasiment anecdotique. En fait, il joue sur le fait que l'immigration, qui était très marginale en Finlande, a très récemment augmenté (en 2015, le nombre de citoyens nés à l'étranger était encore de $4 \%$ contre $7 \%$ aujourd'hui), ce qui a provoqué des réactions assez négatives de la part d'un peuple qui avait très peu de contacts avec des étrangers jusqu'alors. Comme les autres partis populistes de droite radicale en Europe, PS a su catalyser les craintes et les rancœurs de segments de la population qui se sent déclassée (Soini désignait son parti comme «un parti de travailleurs sans socialisme »), non adaptée à la mondialisation, et concurrencée par l'arrivée d'étrangers sur son sol ${ }^{33}$. De plus, il ne cesse d'agiter l'épouvantail d'autres pays européens : la Suède, la France, la Grande-Bretagne sont décrites comme des catastrophes, l'immigration y ayant engendré des "ghettos ", des crises sociales et du chômage. La crise migratoire récente a été une opportunité pour renforcer ce discours. Avec Halla-Aho, le parti, qui se désigne dans son programme de principe de 2018 comme un parti «du franc-parler ${ }^{34}$ ", est devenu plus hostile à l'immigration et plus ouvertement raciste (durant la campagne de 2019, il a largement exploité une affaire d'abus sexuel ayant impliqué des immigrés à Oulu ${ }^{35}$ ). Sous Soini, PS faisait une distinction entre une immigration qui serait une charge pour le pays, et une immigration profitable, en raison du manque de main-d'œuvre en Finlande. Sous HallaAho, l'accent est plutôt mis sur le fait que l'immigration "n'amène pas de nouveaux contribuables en Finlande » et que de ce fait «le retour des migrants dans leur pays d'origine est le plus important... Le parti des Finlandais doit stopper toute l'immigration nuisible qui a été si coûteuse et dommageable pour la société finlandaise ${ }^{36} »$. (Ce qui se prolonge par des allusions à une montée de la criminalité et de l'insécurité liée à l'immigration).

L'argumentaire anti-immigration de Ps est lié à un "chauvinisme d'Étatprovidence $^{37}$ » : pour PS, « le patriotisme c'est un égoïsme au service de la communauté finlandaise ${ }^{38} »$. Selon le parti, il faut à tout prix éviter que les immigrés ne viennent pour profiter des conditions de vie généreuses offertes par l'État-providence. Timo Soini prenait soin de ne pas critiquer l'immigration en soi, mais les mauvaises politiques d'immigration, et disait que s'il fallait diminuer l'immigration, c'était pour que les immigrés aient de bonnes conditions de vie... et qu'ils réussissent leur assimilation à la société finlandaise sans mettre en péril la culture finnoise. Alors que sous Soini, l'objectif de PS était une politique d'assimilation ne tolérant pas d'échec (le parti prévoyait différents cas de renvoi des étrangers), sous Halla-Aho, il est davantage question de stopper toute immigration "dommageable». De plus, l'accent est mis sur l'attribution des ressources aux Finlandais les plus modestes. En 2019, la liste de ses priorités était la suivante: "Maintien de bons services sociaux, de bons salaires et conditions de travail, de bonnes écoles, de bonnes attitudes sociales incluant le respect de l'égalité et du consensus social et d'une manière générale, une bonne Finlande ", ceci s'opposant au gaspillage, par les gouvernements précédents de «milliards » dépensés pour " l'immigration et l'amélioration du monde » (alors qu'il est urgent de réparer des "écoles infectées par de la moisissure et de l'air viciée ${ }^{39}$ ». En matière sociale, plus généralement, PS reproche aux partis classiques d'avoir mal géré l'État finlandais. Il propose des mesures visant à redresser les finances publiques, tout en assurant la sécurité et le bien-être du citoyen finlandais ordinaire. PS affirme la nécessité de 
maintenir les aides aux personnes âgées, aux handicapés, aux familles modestes. La solution est toute trouvée : les coupes budgétaires doivent résulter d'une diminution des aides aux immigrés et à l'aide internationale au développement. Toutefois, la conception qu'a le parti de l'État-providence est particulière : il souhaite renforcer la responsabilité personnelle du citoyen. Cet élément, déjà présent sous la direction de Soini, semble se renforcer avec J. Halla-Aho. S'il a pour objectif une réduction du coût de la vie pour les plus modestes, le programme de principe de 2018 affirmait que « [1]e parti est opposé à l'attitude selon laquelle le gouvernement est toujours là pour fournir ce qui manque. Le parti est favorable à des services fondamentaux fournis par le gouvernement lorsque les impôts sont raisonnables et utilisés de façon compétente au bénéfice des Finlandais ${ }^{40}$ ». Par ailleurs, le programme électoral de 2019 affirme que « le présent et le futur du bien-être des citoyens finlandais nécessitent la promotion et le soutien du travail productif et d'un entrepreneuriat efficace ${ }^{41} »$. Si certains ont cru voir dans ce volet social un programme de gauche visant à conserver le modèle nordique, il mélange une approche très sociale, mais chauvine de l'État-providence à des mesures très libérales, ce qui est typique des partis populistes de droite radicale.

Le contenu de la politique d'immigration voulue par PS s'est précisé lors du passage de PS au gouvernement Sipilä. Une série de mesures fut annoncée au sujet de l'immigration: les immigrés devaient être pris en charge dans des "programmes d'intégration » obligatoires, avec cours de finnois et aide à la recherche d'emploi, mais on leur attribuerait des aides sociales inférieures à celles accordées aux citoyens finlandais. Les critères de la réunion familiale devaient être durcis, l'expulsion des immigrés délinquants accélérée. En 2016, le ministère des Affaires étrangères, dirigé par Timo Soini, avait communiqué sur les réseaux sociaux afin de décourager les demandeurs d'asile potentiels de postuler en Finlande. Réforme Bleue, qui apparaissait plus modéré au début a également été en ce sens avec quelques propositions faites en vue de la campagne des législatives de 2019. Ces initiatives ne sont pas sans rappeler la politique danoise, qui, sous la houlette du Parti du peuple danois, est devenue l'une des politiques d'immigration les plus dures en Europe ${ }^{42}$.

Les positions prises par le parti des Finlandais amènent à évoquer renvoient à la question du racisme. Nombre de ses membres, dont les plus éminents ont publiquement tenu des propos racistes sans en être exclus. Jussi Halla-aho ${ }^{43}$ et Olli Immonen ont tenu des propos insultants sur l'Islam (« religion de pédophiles ${ }^{44}$ » et "d'idéologie totalitaire et fasciste $\left.{ }^{45} »\right)$. Halla-aho a été condamné par la Cour suprême pour « troubles contre les cultes religieux » et « agitation ethnique ». Olli Sademies, élu à Helsinki, a quant à lui suggéré de stériliser les noirs, parce qu'ils ont trop d'enfants, ceci afin d'éviter un naufrage de l'aide sociale... Il n'a pas été lui non plus exclu du parti. Les sanctions semblent plus fréquentes pour des propos pro-nazis ou fascistes que pour des propos racistes: Jussi Halla-aho a été exclu pour deux semaines de l'Eduskunta (le parlement finlandais) pour avoir déclaré que «ce qu'il faudrait à la Grèce, c'est une junte militaire ». Et l'un des rares cas d'exclusion de PS est celui de James Hirvisaari, qui s'est fait photographier en faisant le salut nazi devant l'Eduskunta. Timo Soini, pour sa part, s'est toujours gardé d'exprimer des propos xénophobes, mais il a eu, à l'évidence, une attitude très laxiste vis-à-vis des membres de son parti, cultivant une grande ambiguité à leur égard. Néanmoins, il a lui-même été mis devant ses contradictions par un journaliste de la $\mathrm{BBC}$, à qui il a expliqué qu'il ne pouvait être derrière chacun des membres de son parti. Sur la question de l'exclusion, sa position était qu'en tant que simple humain il ne pouvait condamner plus que la justice ${ }^{46}$. 
Finalement, Timo Soini trouva un moyen de couper court aux accusations de racisme qui lui était régulièrement faites : il boycottait les journaux qui l'accusaient. De son côté, Halla-Aho a un profil plus tranché. Dans son blog appelé Scripta, dont le thème est : "l'immigration, le multiculturalisme, la tolérance, le racisme, la liberté de parole et le politiquement correct", qui compte entre 3000 et 6000 lecteurs par jour, il exprime des idées très critiques envers l'immigration et le multiculturalisme. Il y a notamment écrit que « la critique d'idéologies totalitaires et fascistes comme l'Islam ne devrait pas être considérée comme du racisme ni ne devrait être considérée comme une infraction $»^{47}$. De nombreux chercheurs ont observé que la montée de PS a coïncidé avec une très nette augmentation des propos racistes sur les réseaux sociaux. De plus, plusieurs membres du parti, dont Halla-Aho, sont membres d'une association ultranationaliste appelée Suomen $\mathrm{Sisu}^{48}$.

La posture ethno-nationaliste de PS s'accompagne de valeurs très conservatrices. PS défend la famille, l'éducation religieuse, la morale et l'ordre. Il souhaite une plus grande sévérité pour punir les criminels : le boxeur Tony Halme, élu à Helsinki, voulait envoyer les pédophiles, les violeurs et les dealers dans les prisons russes... Par ailleurs, sous Soini, PS a proposé une politique d'éducation très rétrograde: il souhaitait favoriser l'enseignement de l'artisanat et du design folklorique à l'école et rendre l'étude du Kalevala obligatoire à l'école; ce recueil de légendes populaires fut compilé par Elias Lönnrot au $19^{\text {ème }}$ siècle afin de promouvoir la culture et la langue finnoise à l'époque de la construction du nationalisme finlandais. Plus «droitières » encore furent les propositions de "mettre davantage l'accent sur l'éducation physique " parce que "c'est une matière concernant à la fois la santé physique et mentale ${ }^{49}$ ", de noter les élèves pour leur conduite, et de réduire le nombre d'admissions dans les universités... Il est vrai que PS fustige les élites et les intellectuels. De même, les derniers programmes du parti mettent l'accent sur les valeurs « finlandaises et occidentales traditionnelles basées sur des fondamentaux chrétiens bien établis ${ }^{50} »$.

En matière de politique étrangère, le programme de PS vise à défendre la souveraineté du pays. PS est hostile à une adhésion à l'OTAN, et à l'intégration européenne. PS est le parti dont la position anti-européenne a été la plus radicale et la plus constante en Finlande. Il accuse l'UE de ne pas être démocratique et de favoriser les intérêts du grand capital. PS souhaite remplacer l'UE par une « une confédération souple d'Etats indépendants travaillant pour le libre-échange et des bénéfices économiques ${ }^{51}$ \%. Le parti est également favorable à un retrait de l'union monétaire et envisage de renégocier l'adhésion à l'Union ${ }^{52}$. Selon lui, les intérêts du peuple finlandais ne sont tout bonnement pas assurés dans le système européen actuel.

\section{La fin de l'exception finlandaise}

L'examen de l'idéologie de PS permet de constater qu'il s'agit bien d'un parti populiste possédant les caractéristiques définies par Jens Rydgren, à savoir ethno-nationalisme, xénophobie, autoritarisme et style populiste. Alors qu'il siège aujourd'hui à droite, les auteurs finlandais ont eu tendance à classer le parti des Finlandais au centre, du fait de sa filiation agrarienne mais aussi pour deux raisons : d'une part ses prises de positions oscilleraient entre droite et gauche part, d'autre part, il serait dépourvu de lien avec des organisations néo-nazies ou néo-fascistes. 
on peut observer que la défense de l'Etat-providence n'est pas une exception parmi les autres partis populistes européens, même de droite. Lorsque Timo Soini définissait son parti comme un "parti ouvrier sans socialisme ", il utilisait quasiment les mêmes mots que Jörg Haider, qui définissait le FPÖ autrichien comme un parti «social, sans socialisme ». De plus, la conception particulière que PS a de l'Etat-providence, selon laquelle l'individu est responsable de son bien-être, est justifiée en termes nationalistes: le programme de 2011 stipulait que «la santé et le bien-être sont les fondements du succès d'une nation ${ }^{53}$ ». Et le parti martèle toujours que les bénéfices de l'Etat-providence finlandais doivent être destinés aux Finlandais... Cette conception est celle d'un «Etat-providence national qui protège les intérêts du Finlandais ordinaire tout en protégeant la souveraineté politique et culturelle de la Finlande ${ }^{54}$ ». Le seul point du programme de PS qui soit nettement «à gauche» est son programme fiscal, visant à décourager la consommation "outrancière » en augmentant les impôts sur les hauts revenus et le capital. Mais cela n'a rien d'étonnant de la part d'un parti qui prend fait et cause pour « le Finlandais ordinaire ».

Sur la question des liens avec des organisations extrémistes, ils n'ont pas été démontrés. Cependant des sympathies se sont exprimées : nous avons évoqué le salut nazi du député James Hirvisaari, nous pourrions aussi mentionner ses propos sur l'admiration qu'il portait à Jörg Haider ou la déclaration d'admiration du député Jurho Eerola pour Mussolini... Fait plus troublant, sur la page d'accueil du blog de Jussi Halla-Aho, se trouve un lien renvoyant vers le site Gates of Vienna, un site donnant la parole à des néo-nazis et tenants de la thèse du grand remplacement entre autres, où écrivait Anders Breivik ${ }^{55}$. Il y avait également un lien vers le blog de Mikko Elillä, aujourd'hui désactivé pour cause d'incitation à la haine racisale ${ }^{56}$. Au total, l'examen du programme de PS ne laisse guère entrevoir de différences significatives avec ceux des autres partis populistes de droite radicale, tels le Rassemblement national en France: leurs positions anti-européenne, nationaliste, anti-immigration et pour le maintien d'une protection sociale étendue sont comparables. Si l'on examine l'électorat de PS, on constate qu'il a le profil-type de l'électorat d'extrême-droite en Europe : les électeurs sont plutôt jeunes, masculins, peu diplômés et se sentent exclus ou déçus par les partis traditionnels. Depuis 2011, cet électorat s'est élargi à une partie de l'électorat socialdémocrate et de l'électorat $\mathrm{du}$ parti de la Coalition nationale, plus les abstentionnistes (1/5ème des électeurs de PS en 2001 s'étaient abstenus aux élections de 2007). Ce profil d'électeurs reste vrai en 2019, même si PS, se présentant aujourd'hui comme le «seul " parti authentiquement conservateur face à des partis représentant diverses facettes du libéralisme, a réalisé de bons scores dans certains quartiers favorisés, en particulier à Helsinki ${ }^{57}$.

Pour toutes ces raisons, il semble bien que la théorie de « l'exception finlandaise » soit périmée. Le succès de PS est en passe de marquer durablement la vie politique finlandaise. Dans un premier temps, les autres partis l'ont considéré comme un parti " comme les autres » et ont, pour le centre et les conservateurs, accepté de siéger avec lui au cabinet Sipilä. Certes, cette période fut celle d'échecs notables pour PS : en termes de politique, ce furent l'échec par exemple de son projet de finnisation de l'éducation, de sa campagne contre la loi pour le mariage pour tous, ou en matière d'immigration puisque durant la crise des migrants, la Finlande dut accueillir son lot de réfugiés, contrairement à ce qu'avait promis PS lors de sa campagne (néanmoins, si ce dossier fut un échec pour Réforme bleue, remplaçant PS au gouvernement après la

Nordiques, 39 | 2020 
scission du parti, il fut très bénéfique à PS passé dans l'opposition). Tout cela semble plaider en faveur de l'argument que les partis populistes en général résistent mal à l'épreuve du pouvoir ${ }^{58}$. Quelques mois après son entrée au gouvernement en 2015 , les sondages indiquaient que les électeurs commençaient à être déçus des concessions faites par le parti au sein de la coalition (en octobre 2015, un sondage indiquait que près de $60 \%$ des électeurs ayant voté pour PS auraient reconsidéré leur vote si les élections s'étaient tenues alors, et qu'une partie de ces votes se serait reportée sur les Sociauxdémocrates $\left.{ }^{59}\right)$.

Pour autant, ce que l'on constate, c'est que le phénomène du populisme radical de droite s'est profondément inscrit dans la vie politique finlandaise. Le discours de PS a brisé le consensus traditionnel et a amplifié l'expression des mécontentements et des craintes envers les évolutions sociétales. Il a libéré la parole xénophobe avec une intensité étonnante et d'une manière qui aurait été impensable il y a une vingtaine d'années. La fulgurante ascension du populisme de droite radicale en Finlande a obligé les autres partis à se positionner vis-à-vis de lui, voire à reprendre ses thèmes. On peut par exemple citer le très remarqué discours du Président de la République, Sauli Niinistö (du parti du Centre), lors de l'ouverture de la session parlementaire de février 2016. Le Président a parlé du phénomène migratoire comme "d'un problème sérieux » mettant en question «l'Europe, la Finlande, le mode de pensée occidentale et nos valeurs » ... « Encore quelques années auparavant, nous exportions nos valeurs et les considérions comme indiscutables, maintenant nous devons nous demander si nous pourrons les préserver ${ }^{60} \%$. Quant à la gauche, en particulier les Sociaux-démocrates, ils ont été obligés de se positionner par rapport à PS.

Le parti des Finlandais est bien parti pour marquer, de façon durable, la scène politique finlandaise par ses idées. Aux législatives de 2019, le nouveau PS, sous la houlette de Jussi Alla-Aho, a raffermi ses thèses sur l'immigration et sur l'Europe (affirmant que l'objectif est de la quitter à plus ou moins long terme, ainsi que de quitter la zone euro). Il a obtenu $17,7 \%$ des voix, soit la seconde place au scrutin après les Sociauxdémocrates. Sa position actuelle, dans l'opposition, lui donne l'avantage de développer sa critique des gouvernants sans endosser d'éventuels échecs politiques... Tout en jouissant d'une liberté de parole lui permettant de pousser le bouchon toujours plus loin hors des limites du droit et de la morale, en particulier sur le thème du racisme. ${ }^{61}$ La Finlande s'était crue immunisée contre le populisme de droite radicale, mais en réalité elle semble emprunter le même schéma que beaucoup de pays européens et elle assiste à un développement auquel elle n'était pas préparée, tant sur le plan des instruments juridiques (la loi anti-discrimination ne date que de 2004), que sur le plan des mouvements sociaux : les associations de lutte contre le racisme, quoiqu'en plein développement, sont encore débutantes, ce pays n'en ayant jamais connu le besoin auparavant. A l'heure où nous écrivons, les sondages indiquent que le parti des Finlandais, depuis plusieurs mois, est le parti ayant le plus fort soutien populaire dans le pays ${ }^{62}$. 


\section{NOTES}

1. Le politologue allemand Herbert Kitschelt écrivait encore en 2007 qu'il était très peu probable qu'une droite radicale se développe en Finlande et en Suède. Herbert Kitschelt, "Growth and Persistence of the Radical Right in Post-Industrial Democracies: Advances and Challenges in Comparative Research », West European Politics, vol. 30, n 5, 2007, p. 1176-1206.

2. Jens Rydgrén, «Introduction », in : Class politics and the radical right, Jens Rydgren (éd), London, Routledge, 2013.

3. Le terme Perussuomalaiset signifie plutôt "les Finlandais ordinaires » ou "typiques ». La traduction par l'expression «Vrais Finlandais» a été jugée vexatoire pour les Finlandais n'appartenant pas à ce parti, c'est pourquoi, en 2011, le parti a décidé de traduire son nom finlandais par "parti des Finlandais». Cf: True Finns name their party "The Finns », Yle, 21/08/2011, http://yle.fi/uutiset/quottrue_finnsquot_name_their_party_quotthe_finnsquot/ 2807245

4. Iso jytky, parfois traduit par «la grosse bombe» dans la presse. Ce terme évoquant une explosion avait été utilisé par le leader du parti des Vrais Finlandais, Timo Soini, pour désigner le phénomène par lequel son parti, un petit parti d'opposition, avait rapidement émergé pour devenir un parti de gouvernement.

5. Dans un contexte particulier opposant un président Niinistö, très populaire, à un ensemble de partis très fragmenté.

6. Aux européennes de 2019, il est arrivé en $4{ }^{\text {ème }}$ position avec $13,8 \%$ des voix.

7. La coalition formée après les élections législatives de 2019, dirigée par Sanna Marin, réunit le parti Social-démocrate, le parti du Centre, la Ligue verte, l'Alliance de gauche, et le parti du Peuple suédois.

8. Le roi Erik de Suède lança des croisades en direction de la Finlande en 1150, la conquête fut achevée en 1323 et la Finlande fit partie intégrante du royaume de Suède jusqu'en 1809; elle devint alors un grand-duché de l'empire russe.

9. Max Engman, «Finns and Swedes in Finland, » in : SvenTägil, (éd.) Ethnicity and Nation Building in the Nordic World, London, Hurst, 1995. Matti Klinge, Från lojalims till rysshat, Stockholm, Ordfront, 1988. Kenneth Mac Rae, Conflict and Compromise in Multilingual Societies. Finland. Helsinki, The Finnish Academy of Science and Letters and Wilfrid Laurier University Press, 1999.

10. L'Ordonnance sur la langue de 1863 déclara que le «finnois est sur un pied d'entière égalité avec le suédois pour toutes les questions concernant directement la partie finnophone de la population".

11. Les premières manifestations de ce clivage politique se situèrent dans les années $1870: a u$ parlement, deux états (noblesse et bourgeoisie) étaient dominés par le Parti suédois et les deux autres (clergé et paysannerie) par les partis représentant des finnophones. Lorsque le suffrage universel fut mis en place en 1906, le parti du Peuple suédois fut créé. Selon P. Pesonen et O. Riihinen, «les disputes linguistiques furent très importantes entre la fin des années 1880 et la fin des années 1930 ». La loi constitutionnelle de 1919 reconnut le suédois et le finnois comme langues officielles $\mathrm{du}$ pays et garantit le droit de chaque citoyen d'utiliser sa propre langue Pertti Pesonen, Olavi Riihinen, Dynamic Finland, The Political System and the Welfare State, Helsinki, Studia Fennica, Historica, 2002.

12. K. Mac Rae, Conflict and Compromise in Multilingual societies, op. cit.

13. Ibid., p. 166.

14. Les suédophones représentent autour de $5 \%$ de la population finlandaise de nos jours.

15. Pekka Kalevi Hämäläinen, Nationalitetskampen och språkstriden i Finland, 1917-1939, Helsingfors : Holger Schildts Förlag, 1969. 
16. Voir par exemple : Lauri Karvonen, Parties, Governments and Voters in Finland, Colchester, ECPR Press, 2014. C'est surtout au cours de son troisième mandat (1968-1974) que la politique de Kekkonen fut autoritaire, le président se posant en seul gardien de l'intérêt national. À ce titre, il mena une diplomatie personnelle avec le Kremlin (par ex. affaire de la négociation de la frontière finno-soviétique en juin 1968), tandis que la politique de sécurité devint pour lui une compétence personnelle. Il parvint aussi à dominer le Parlement et la vie politique (avec par exemple l'éviction du parti conservateur de la Coalition nationale des gouvernements entre 1966 et 1987 pour des «motifs généraux ». Pour plus d'exemples, cf. David Arter, Scandinavian Politics Today, Manchester University Press, 1999, pp. 332 s. De nombreux débats et controverses ont eu lieu, après la guerre froide, sur le rôle de Kekkonen dans la politique finlandaise. Cf. Jason Lavery, " All of the Presidents' historians: The Debate over Urho Kekkonen ", Scandinavian Studies, vol. 75, $\mathrm{n}^{\circ}$ 3, Fall 2003, pp. 375-398.

17. Johanna Korhonen, «Ten Paths to populism, How Silent Finland Became a Playing Field for Loud Populism ", in: Populist Fantasies - European Revolts in Context, C. Fieschi, M. Morris, L. Caballero (eds), Counterpoint, September 2012, p. 220.

18. Le taux de pénétration d'Internet en Finlande était en 2019 de $94 \%$, soit l'un des plus élevés en Europe. Internet World Stats, https://www.internetworldstats.com/stats4.

19. La toute-puissance du Président Kekkonen (avec l'appui de Moscou) était telle qu'en 1973 une loi d'urgence étendit ses pouvoirs pour quatre années supplémentaires, ce que le Président justifiait en disant que s'il ne restait pas au pouvoir, les Soviétiques n'accepteraient pas que la Finlande signe le traité de libre-échange qu'il était en train de négocier avec la CEE. Aux élections de 1978, le candidat Kekkonen, ayant obtenu le soutien de neuf partis, se fit réélire en refusant de débattre avec ses opposants.

20. Tapio Raunio, "The Finns: Filling a Gap in the Party System », in: Exposing Demagogues. RightWing and National Populist parties in Europe, K. Grabow,F. Hartleb (eds), Berlin, Center for European Studies, Konrad Adenauer Stiftung, 2013.

21. Alain,Lefèvre, "La Finlande dans la crise et l'évolution de son modèle social ", Nordiques, $\mathrm{n}^{\circ} 23,2010$, p. 27-39.

22. Statistics Finland, Income Distribution Statistics, 2007, 2008, 2009.

23. Les Finlandais votent à la fois pour des partis et pour des noms.

24. La coalition arc-en-ciel du gouvernement Lipponen II réunissait les Sociaux-démocrates, l'Alliance de gauche, le parti de la Coalition nationale, la Ligue verte, et le parti populaire suédois. 25. J. Jokela, K. Korhonen, « No longer a model pupil in European politics? Finland's EU policy after the general elections", Commentary, $28^{\text {th }}$ April 2011, $\mathrm{n}^{\circ}$ 6. European Policy Institutes Network. https://epin.org/

26. Les responsables de ce parti avaient «omis » de déclarer toutes leurs sources de financement pour la campagne de 2007 (ce qui est obligatoire mais non sanctionné par la loi).

27. The Finns Party, Principle Program, 19/10/2018. https://www.perussuomalaiset.fi/wpcontent/uploads/2019/06/Periaateohjelma-19.10.2018_SU_In-English.pdf.

La question était également très développée dans le programme de 2007.

28. The Finns Party, Finns Party - Elections 2015. The Main concerns. https:// www.perussuomalaiset.fi/kielisivu/in-english/

29. L'article 17 de la constitution finlandaise de 2000 stipule que «les langues nationales de la Finlande sont le finnois et le suédois ». Elle garantit le droit de chacun à utiliser sa propre langue devant les administrations, et accorde également aux Sames et aux Roms, le droit de développer leur propre langue et culture.

30. Voir les propos de la sociologue Anna Rastas et de l'ombudsman contre les discriminations Kirsi Pimiä in: «Researcher: Populists use misleading immigration language to their advantage ", Helsinki Times, 30/07/2015. 
31. Perussuomalaisten eduskuntavaaliohjelma, 2007. https://www.perussuomalaiset.fi/tietoameista/puolueohjelma/

32. The Finns party, The Finns Party Principle Program, 19/10/2018. https:// www.perussuomalaiset.fi/wp-content/uploads/2019/06/Periaateohjelma-19.10.2018_SU_In-

English.pdf.

33. Jussi Halla-Aho se positionne davantage comme le seul vrai parti de droite et est parvenu à attirer des électeurs plus urbains et plus favorisés.

34. The Finns Party, Principle Program, 19/10/2018.

35. L'affaire a sa page Wikipedia: https://en.wikipedia.org/wiki/ Oulu_child_sexual_exploitation_scandal

36. The Finns Party, Vote Back Finland, Election program 2019.

37. Le welfare state chauvinism, concept difficilement traduisible, désigne le fait de réserver les prestations sociales de l'Etat-providence à une catégorie de la population, notamment définie sur des bases ethniques, au lieu de les attribuer selon des principes égalitaristes et universalistes.

38. Fit for the Finns - The Finns's party election programme for 2011 - summary. https:// www.perussuomalaiset.fi/wp-content/uploads/2013/04/

Perussuomalaisten_eduskuntavaaliohjelma_2011-english_summary_2.0.pdf

39. Vote Finland Back, op. cit.

40. The Finns party Principle Program, 19/10/2018, op. cit.

41. Ibid.

42. Blanc-Noël, Nathalie, «Régner sans gouverner - ou presque : le cas du parti populiste danois, un populisme établi », Politeia, n²4, automne 2013.

43. Jussi Halla-aho est député européen et député à l'Eduskunta.

44. Propos tenus sur Scripta, le blog de Jussi Halla-Aho, en juin 2008. http://www.halla-aho.com/ scripta/

45. « Toisinajattelijana Suomessa - Policyn haastattelussa Jussi Halla-aho », Policy 1/2008, p. 16.

46. «BBC Hard Talk grills Timo Soini on Racism », Feb. 2013, https://www.youtube.com/watch? $\mathrm{v}=2$ tqn9BrpEkA

47. «Toisinajattelijana Suomessa - Policyn haastattelussa Jussi Halla-aho » Policy 1/2008, p. 16, Helsingin yliopiston Valtio-opin Opiskelijat ry. Voir aussi : Soeren Kern, « Muslim Immigration Transforms Finland», 28 April 2011, Gatestone Institute, http://www.gatestoneinstitute.org/ 2075/finland-muslim-immigration. On peut trouver sur Wiki quote un florilège de déclarations racistes, mais aussi pro-nazies écrites par Jussi Halla-Aho sur le blog Scripta. M. Halla- Aho publie également des textes sur le blog d'extrême-droite Gates of Vienna, sur le blog Multicultural discourse in Finland and Sweden et sur divers blogs extrémistes, tels Homma, un blog anti-immigration. Le terroriste et néo-nazi Anders Breivik, auteur de la tuerie d'Utøya en Norvège en 2011 avait cité Jusssi Halla-Aho comme une source d'inspiration idéologique... A noter qu'un des créateurs du blog Homma, Mattias Turkkila, est devenu rédacteur en chef du journal du parti PS, Perussuomalainen.

48. Cette association se dit nationaliste et patriote. Elle est opposée à l'immigration, au multiculturalisme, défend la souveraineté de l'Etat et s'oppose à la supranationalité. Les députés Olli Immonen, James Hirvisaari et Juho Eerola, élus en 2011, en sont membres. Jussi Halla-Aho en a démissionné en 2019. Jutila, Karina, Sundell, Björn, « The Populism of the Finns Party, Fun or Ugly?» E2 Think tank, http://archive.gef.eu/uploads/media/ Full_Article_The_Populism_of_The_Finns_Party_-_Fun_or_Ugly.pdf. Arter, David, «The Breakthrough of Another West European Populist Radical Right Party? The Case of the True Finns ", Government and Opposition, vol. 45, n 4, 2010, p. 484-504. Simo Grönroos, Suomen Sisun 10vuotishistoriikki, Helsinki, Suomen Sisu, 2008. https://www.suomensisu.fi/esittely/

49. Fit for the Finns - The Finns party's election programme for the parliamentary election 2011, op.cit. 
50. The Finns' party European Union policy, 21/1/2019. https://www.perussuomalaiset.fi/wpcontent/uploads/2019/05/EUpolitiikkaENG_2019.pdf

51. Ibid.

52. Ibid.

53. Fit for the Finns, op. cit.

54. Johan Nordensvald, Markus Ketola, «Nationalist reframing of the Finnish and Swedish Welfare State : the Nexus of Nationalism and Social Policy in Far-right Populist parties », Social Politics and Administration, vol. 49, $\mathrm{n}^{\circ} 3$, May 2015.

55. https://www.halla-aho.com/scripta/johdanto_uudelle_lukijalle.html (consulté le 26/06/2020).

56. http://mikkoellila.thinkertothinker.com/ (consulté le 26/06/2020). Voir aussi note 44.

57. Kimmo Grönlund, Kim Strandberg, eds. Voting and public opinion in Finland, The parliamentary election of 2019, Åbo Akademi, Institutet för samhällsförskning, 2019. https://www.abo.fi/wpcontent/uploads/2019/07/Voting-and-Public-Opinion-in-Finland.pdf

58. Nathalie Blanc-Noël, «When governing is losing advantage. Denmark and Finland, two opposite cases of radical right parties' influence on policy making", in: Do They Make a Difference? The Policy Influence of Radical Right Populist Parties in Western Europe, B. Biard, L. Bernhard, H-G. Betz, ECPR Press/Rowman and Littlefield International, 2019.

59. "Fewer than half voters would recast their vote for Finns party ", Helsingin Sanomat, 23 oct 2015.

60. Sauli Niinistö jyrähti: «Polttopullo rakennukseen on minulle vieläkin murhapoltto », Uusi Suomi, 1/1/2016.

61. N. Blanc-Noël, « When Governing is losing advantage »... op. cit.

62. Plus d'un cinquième des électeurs $(21,7 \%)$ ont exprimé leur soutien à PS et ces mêmes sondés sont aussi ceux qui sont le plus sûrs de leur choix, déclarant à $61 \%$ être « absolument certains » de voter pour PS. «Finns Party leads way, support for ruling parties at $53 \%$ », 13/02/2020, Alma Media, https://www.helsinkitimes.fi/finland/finland-news/politics/17326-alma-media-finnsparty-leads-way-support-for-ruling-parties-at-53.html

\section{RÉSUMÉS}

Jusqu'au début du $21^{\text {ème }}$ siècle, la Finlande passait pour une exception politique en Europe, n'étant pas concernée par la montée du populisme de droite radicale. Cette situation changea du tout au tout au cours des années 2010, en particulier lorsque le parti des Finlandais obtint le second meilleur score aux élections législatives de 2011. Depuis lors, ce parti constitue l'une des toutes premières forces politiques dans ce pays. Cet article explore ses origines historiques ainsi que son idéologie, du Parti rural fondé par Veikko Vennamo dans les années 1960 jusqu'au parti des Finlandais et à Réforme Bleue aujourd'hui.

Until the beginning of the 21st Century, Finland was said to be a political exception in Europe, as this country seemed to be untouched by the rise of radical right-wing populism. This situation dramatically changed during the first decade of the Century, especially in 2011, when the Finns party obtained the second best score at the parliamentary election. Since that time, the Finns have been one of the major political forces in Finland. This paper explores the historical origins 
of the party and its ideology, from the Finnish rural party founded in the 1960's by Veikko Vennamo to the present Finns and Blue reform parties.

INDEX

Mots-clés : parti politique, populisme, Finlande

Keywords : political party, populism, Finland

\section{AUTEUR}

NATHALIE BLANC-NOËL

maître de conférences en Science politique, directrice-adjointe de l'IRM, Université de Bordeaux. 